\title{
Metabolic consequences of antihypertensive therapy
}

\author{
JEFFREY W. OLIN, D.O.
}

Cleveland, Ohio

In addition to

hypertension, metabolic abnormalities such as elevated serum lipid levels, glucose intolerance, and hypokalemia, increase risk for adverse cardiovascular events. A number of commonly used antihypertensive drugs produce biochemical changes that increase the prominence of one or more of the additional risk factors as they lower blood pressure. Thiazide diuretics, for example, produce dose-related elevations in serum cholesterol and glucose values, as well as significant decreases in serum potassium. Although beta-blockers do not clinically affect potassium metabolism significantly, they do raise both cholesterol and glucose levels. The angiotensin-converting enzyme inhibitor captopril is the first of a new class of antihypertensive agents that lower blood pressure as effectively as previous therapies without adversely affecting serum levels of total, highdensity lipoprotein, and low-density lipoprotein cholesterol or triglyceride, glucose, or potassium levels. In addition, captopril has been shown to blunt the adverse metabolic consequences of diuretic therapy when the two agents are administered concomitantly. It appears that the stepped-care approach is now too simplistic and that the design of antihypertensive regimens in the 1980 s should be approached judiciously and on a more individualized basis.

The classic approach to antihypertensive therapy, as recommended by the Joint National Committee on Detection, Evaluation, and Treatment of High Blood Pressure, ${ }^{1}$ utilizes a steppedcare framework in which treatment is begun with either a thiazide diuretic or a beta-blocking agent. If the first type of drug fails to control blood pressure adequately, the second type is added to the therapy. If blood pressure is still not well controlled, a vasodilator is added as a step-three medication. While this treatment plan may be effective in lowering blood pressure, it discourages individualization of the antihypertensive regimen and does not fully consider the problems introduced by various therapies. In many patients, the price for lowering elevated blood pressure is an increase in other cardiovascular risk factors.

Elevated lipid levels, glucose intolerance, and hypokalemia as cardiovascular risk factors

The Framingham Study ${ }^{2}$ demonstrated that cardiovascular morbidity and mortality are directly related to total cholesterol levels. In the Honolulu Heart Program, ${ }^{3}$ fatal coronary heart disease was almost twice as prevalent in patients with elevated serum cholesterol values as in random sample patients; the incidence of fatal coronary heart disease was more than two times higher for patients with elevated serum triglyceride levels as for the random sample group. The Framingham data ${ }^{2}$ also indicated that the incidence of coronary heart disease is inversely related to the level of high-density lipoprotein (HDL) cholesterol, and, indeed, HDL cholesterol may be the single most powerful determinant of cardiovascular risk.

Glucose intolerance is a second identified risk factor for cardiovascular disease. In the Whitehall study, ${ }^{4}$ investigators demonstrated that glucose intolerance and diabetes mellitus pose a heightened risk for the development of coronary heart disease. Their data also suggested that hypertension was extremely important as a cardiovascular risk factor in these patients. Glucose intolerance also has been correlated with raised serum cholesterol and triglyceride values and may increase risk secondarily by raising blood lipid levels. ${ }^{5}$

A decreased serum potassium level is a third metabolic risk factor for adverse cardiovascular events. Asymptomatic hypokalemia may place the patient at increased risk for sudden cardiovascular death, ${ }^{6}$ especially in the presence of pre-existing electrocardiographic (EKG) abnormalities. The Multiple Risk Factor Intervention Trial Research Group ${ }^{7}$ found that hypertensives with resting EKG 
abnormalities were at greater risk for sudden coronary heart disease deaths than were hypertensives without such phenomena. The exact reason for this is unclear.

\section{Antihypertensive agents and serum lipid values}

The antihypertensive agents that are most clearly implicated in producing elevated serum lipid levels are thiazide diuretics and beta-blockers. In a double-blind crossover study ${ }^{8}$ comparing hydrochlorothiazide, chlorthalidone, and placebo in patients with mild hypertension, mean plasma cholesterol increases of $14.8 \pm 3.0 \mathrm{mg} . / \mathrm{dl}$. and $18.8 \pm$ $3.6 \mathrm{mg}$./dl. were documented for patients taking hydrochlorothiazide and chlorthalidone, respectively. Increases in triglyceride levels were even more marked. This represents a clear trade-off, in that blood pressure was effectively lowered at the expense of increases in blood lipid levels. To illustrate, accumulated data ${ }^{9}$ suggest that a 50 -year-old male nonsmoker with a diastolic blood pressure of $100 \mathrm{~mm} . \mathrm{Hg}$ and a serum cholesterol level of 202 $\mathrm{mg} . / \mathrm{dl}$. has a 3.2 percent risk of myocardial infarction within 5 years and an 18 percent risk within 20 years. If a thiazide diuretic were given without adequate dietary control and the diastolic blood pressure decreased to $90 \mathrm{~mm}$. Hg while the serum cholesterol value rose to $238 \mathrm{mg}$./dl., the 5 -year risk probability is still 3.2 percent, while the 20 -year risk is 17 percent. Even though blood pressure would be lowered, there would be little change in the overall cardiovascular risk profile. Similar risk factor balancing may underlie the failure of some studies $^{10}$ to demonstrate significant decreases in coronary mortality of patients with mild hypertension.

Elevated levels of total serum cholesterol, lowdensity lipoprotein (LDL) cholesterol, and triglycerides increase the risk of coronary artery dis-

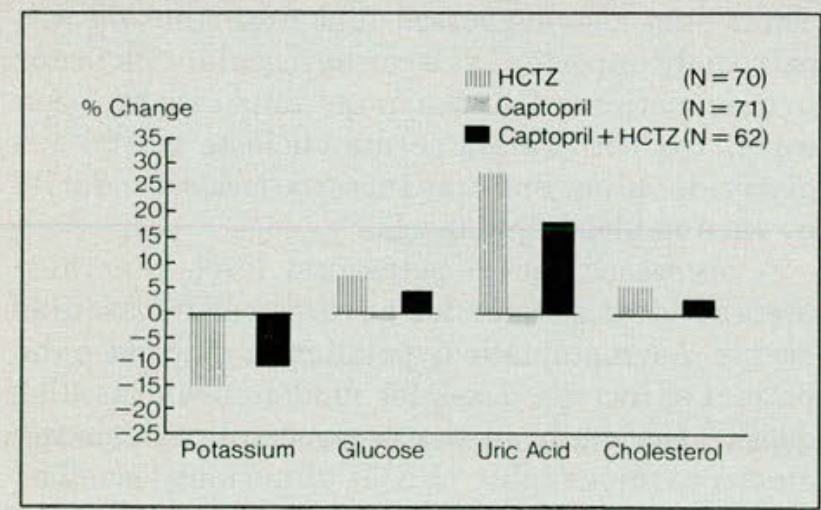

Fig. 1. Effects of hydrochlorothiazide, captopril, and both in combination. (Adapted from Weinberger, ${ }^{20}$ with permission.) ease; HDL cholesterol, in contrast, aids in removing lipids from tissues. ${ }^{11}$ Generally, betablockers (except pindolol, acebutolol and labetalol) have been shown to increase triglycerides and to decrease HDL cholesterol.11,12 The Australian Risk Factor Prevalence Study ${ }^{13}$ showed that metoprolol produces a significant decrease in HDL cholesterol levels, a significant elevation in serum triglyceride values, and a significant increase in the ratio of total cholesterol to HDL cholesterol.

The centrally-acting antihypertensive agents do not seem to raise total cholesterol levels. ${ }^{14}$ However, methyldopa has been shown to reduce HDL cholesterol levels significantly and to elevate the ratio of total cholesterol to HDL cholesterol. ${ }^{15}$ The alphaadrenergic drug guanabenz appears to decrease total serum cholesterol. ${ }^{16}$ Agents not known to alter serum lipid profiles or decrease serum lipid levels include prazosin ${ }^{11,17}$ and calcium entry blockers. ${ }^{18,19}$ Among vasodilators, hydralazine and minoxidil are direct-action agents that have no effect on lipid levels. ${ }^{11}$ However, they stimulate the sympathetic nervous system, causing tachycardia, and they also may cause significant salt and water retention. Thus, a diuretic and/or a beta-blocker frequently must be used with one of these two vasodilators, thereby altering lipid metabolism.

Captopril, an angiotensin-converting enzyme (ACE) inhibitor, does not alter total cholesterol (Fig. 1). ${ }^{20}$ Moreover, when captopril is added to thiazide diuretic therapy, the diuretic-associated rise in serum cholesterol may be effectively blunted. ${ }^{21}$

\section{Antihypertensive agents and glucose tolerance}

Thiazide diuretics cause increases in blood glucose levels that are generally reversible when therapy is discontinued. ${ }^{22}$ Similarly, increases in both fasting blood sugar and 2-hour postprandial blood glucose values have been demonstrated in patients receiving propranolol monotherapy. ${ }^{23}$ Beta-blockers also produce a decrease in serum insulin; this effect is observed with both cardioselective and noncardioselective agents. ${ }^{24,25}$ Labetalol, which has alpha-blocking as well as beta-blocking activity, has no apparent effect on serum glucose values. ${ }^{26}$

Among centrally-acting agents, clonidine has been associated with early reductions in glucose tolerance, ${ }^{27}$ while guanabenz has not. ${ }^{28}$ The peripheral alpha-blocker prazosin increased plasma glucose in a short-term trial, ${ }^{29}$ but not on longer administration. ${ }^{30}$ The ACE inhibitor captopril has no effect on serum glucose, as confirmed in patients treated for several years. ${ }^{31}$ In addition, the expected rise in blood glucose levels following administration of a thiazide diuretic is often blunted by cap- 
topril during combination therapy ${ }^{20}$ (Fig. 1).

\section{Antihypertensive agents and hypokalemia}

Hypokalemia continues to be one of the more worrisome derangements observed during diuretic therapy. Studies by Hollifield and Slaton ${ }^{7}$ and Holland and Associates ${ }^{32}$ point toward higher risk of cardiac arrhythmias during periods of diuretic-induced hypokalemia. Most other antihypertensive drugs have not been associated with low-potassium levels. Captopril does not affect serum potassium levels and lessens the degree of hypokalemia produced by concomitantly administered diuretics (Fig. 1). ${ }^{20}$ Similar effects on serum potassium, as with lipid and glucose levels, may be anticipated with the ACE inhibitor enalapril. Potassium-sparing diuretics and dietary potassium supplements generally are unnecessary during ACE inhibitor therapy, therefore, except for patients in whom hypokalemia has been recorded.

\section{Conclusion}

In summary, all of the available antihypertensive agents effectively lower elevated blood pressure. However, many of these agents have concurrent adverse effects on other known cardiovascular risk factors, including lipid profiles, glucose tolerance, and potassium metabolism. The impact of such antihypertensive medications on cardiovascular morbidity and mortality may be substantially negated by their secondary results. The addition of an ACE inhibitor, which does not produce such side effects, to the antihypertensive armamentarium makes it possible to set aside the outdated constraints of the stepped-care approach and to formulate antihypertensive therapy on an individualized basis.

1. The Joint National Committee on Detection, Evaluation, and Treatment of High Blood Pressure: The 1984 report. JAOA 83:642-59, May 84 2. Kannel, W.B., Castelli, W.P., and Gordon, T.: Cholesterol in the prediction of atherosclerotic disease. New perspectives based on the Framingham Study. Ann Intern Med 90:85-91, Jan 79

3. Reed, D., Yano, K., and Kagan, A.: Lipids and lipoproteins as predictors of coronary heart disease, stroke, and cancer in the Honolulu Heart Program. Am J Med 80:871-8, May 86

4. Fuller, J.H., et al:: Mortality from coronary heart disease and stroke in relation to degree of glycaemia. The Whitehall study. Br Med J 287:867-70, 24 Sep 83

5. Ames, R.P.: Coronary heart disease and the treatment of hypertension. Impact of diuretics on serum lipids and glucose. J Cardiovasc Pharmacol 6(S3):S466-73, 1984

6. Hollifield, J.W., and Slaton, P.E.: Thiazide diuretics, hypokalemia, and cardiac arrhythmias. Acta Med Scand 209:67-73, 1981

7. Multiple Risk Factor Intervention Trial Research Group: Multiple risk factor intervention trial. Risk factor changes and mortality results. JAMA 248:1465-77, 24 Sep 82

8. Grimm, R.H., et al.: Effects of thiazide diuretics on plasma lipids and lipoproteins in mildly hypertensive patients. A double-blind controlled trial. Ann Intern Med 94:7-11, Jan 81
9. Grimm, R.H., and Hunninghake, D.B.: Lipids and hypertension. Implications of new guidelines for cholesterol management in the treatment of hypertension. Am J Med 80 (S2A):56-63, 14 Feb 86

10 Veterans Administration Cooperative Study Group on Antihypertensive Agents: Effects of treatment on morbidity in hypertension. II. Results in patients with diastolic blood pressure averaging 90 through 114 mm. Hg. JAMA 213:1143-50, 17 Aug 70

11. Weinberger, M.H.: Antihypertensive therapy and lipids. Evidence, mechanisms, and implications. Arch Intern Med 145:1102-5, Jun 85

12. Lehtonen, A.: The effect of acebutolol on plasma lipids, blood glucose, and serum insulin levels. Acta Med Scand 216:57-60, 1984

13. MacMahon, S.W., and MacDonald, G.J.: Antihypertensive treatment and plasma lipoprotein levels. The association in data from a population study. Am J Med 80(S2A):40-7, 14 Feb 86

14. Distler, A., Kirch, W., and Lüth, B.: Antihypertensive effect of guanfacine. A double-blind cross-over trial compared with clonidine. Br J Clin Pharmacol 10:49S-53S, 1980

15. Ames, R.P., and Hill, P.: Antihypertensive therapy and the risk of coronary heart disease. J Cardiovasc Pharmacol 4(S2):S206-12, 1982

16. Walker, B.R., Schneider, B.E., and Gold, J.A.: A two-year evaluation of guanabenz in the treatment of hypertension. Curr Ther Res 27:784-96, Jun 80

17. Neusy, A-J., and Lowenstein, J.: Effects of prazosin, atenolol, and thiazide diuretic on plasma lipids in patients with essential hypertension. Am J Med 80(S2A):94-9, 14 Feb 86

18. Midtbø, K., Hals, O., and van der Meer, J.: Verapamil compared with nifedipine in the treatment of essential hypertension. J Cardiovasc Pharmacol 4:S363-8, 1982

19. Wada, S., Nakayama, M., and Masaki, K.: Effects of diltiazem hydrochloride on serum lipids. Comparison with beta-blockers. Clin Ther 5:163-73, 1982

20. Weinberger, M.H.: Comparison of captopril and hydrochlorothiazide alone and in combination in mild to moderate essential hypertension. $\mathrm{Br}$ J Clin Pharmacol 14:127S-31S, 1982

21. Weinberger, M.H.: Influence of an angiotensin converting-enzyme inhibitor on diuretic-induced metabolic effects in hypertension. Hypertension 5(S3): $132-8$, Sep-Oct 83

22. Ames, R.P., and Hill, P.: Improvement of glucose tolerance and lowering of glycohemoglobin and serum lipid concentrations after discontinuation of antihypertensive drug therapy. Circulation 65:899-904, May 82 23. Cressman, M.D., et al.: Glucose tolerance during chronic propranolol treatment. J Clin Hypertension 1:138-44, 1985

24. Carr, A.A.: Hypertension, Rx. Diuretic or beta-blocker first? Modern Med 52:124-31, Oct 84

25. Waal-Manning, H.J., and Bolli, P.: Atenolol $v$ placebo in mild hypertension. Renal, metabolic and stress antipressor effects. Br J Clin Pharmacol 9:553-60, 1980

26. Michelson, E.L., et al.: Multicenter clinical evaluation of long-term efficacy and safety of labetalol in treatment of hypertension. Am J Med 75:68-80, 17 Oct 83

27. Pagani, G., et al.: Comparison of the effects of short-term treatment with guanfacine and clonidine on glucose metabolism, plasma, renin activity and some anterior pituitary hormones. Curr Ther Res 36:155-62, Jul 84

28. Walker, B.R., Hare, L.E., and Deitch, M.W.: Comparative antihypertensive effects of guanabenz and clonidine. J Int Med Res 10:6-14, 1982 29. Barbieri, C., et al.: Metabolic effects of prazosin. Clin Pharmacol Ther 27:313-6, Mar 80

30. Barbieri, C., et al.: Metabolic effects of chronic prazosin treatment. Horm Metab Res 12:331-4, 1980

31. Groel, J.T., et al.: Long-term antihypertensive therapy with captopril. Hypertension 5(S3):145-51, Sep-Oct 83

32. Holland, O.B., Nixon, J.V., and Kuhnert, L.: Diuretic-induced ventricular ectopic activity. Am J Med 70:762-8, Apr 81

At the time this paper was written, Dr. Olin was director of the Hypertension Clinic, Western Pennsylvania Hospital, and clinical instructor of medicine, University of Pittsburgh. He is now an associate staff member in the Department of Peripheral Vascular Disease, Cleveland Clinic Foundation, Cleveland, Ohio.

Dr. Olin, Cleveland Clinic Foundation, 9500 Euclid Avenue, Cleveland, Ohio 44106. 